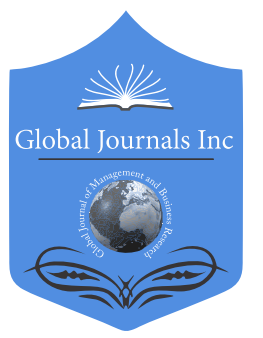

GLOBAL JOURNAL OF MANAGEMENT AND BUSINESS RESEARCH: A ADMINISTRATION AND MANAGEMENT

Volume 20 Issue 15 Version 1.0 Year 2020

Type: Double Blind Peer Reviewed International Research Journal

\title{
Risk Prevention Mechanisms in Asymmetrical Inter-Company Situations: An Exploratory Study in Smes in the BTP in Cameroon
}

\author{
By Alain Marcel Manga Menyomo \& Nicole Patricia Tjani
}

Abstract- While it is true that asymmetrical inter-firm relations offer many opportunities to the various partners and to the SME in particular, they are nonetheless the source of a number of risks. So, how do SMEs protect themselves from the risks associated with inter-firm relations? To answer this question, we have adopted a methodology based on a qualitative approach based on semi-directive interviews with four (04) SMEs in the construction industry. In order to identify and classify the different risk prevention mechanisms mobilized by SMEs. The results obtained show that these SMEs mobilize both contractual and extra-contractual mechanisms. However, they give more priority to extracontractual or relational mechanisms in the prevention of risks.

Keywords: asymmetrical inter-company relations, risk prevention mechanisms, risks, construction and public works, SMEs.

GJMBR-A Classification: JEL Code: M19

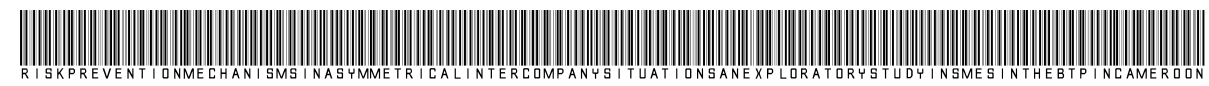

Strictly as per the compliance and regulations of:

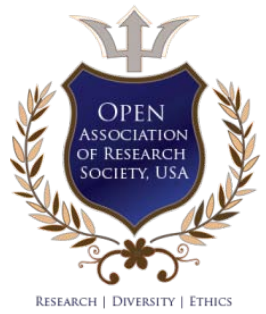

(C) 2020. Alain Marcel Manga Menyomo \& Nicole Patricia Tjani. This is a research/review paper, distributed under the terms of the Creative Commons Attribution-Noncommercial 3.0 Unported License http://creativecommons.org/licenses/by-nc/3.0/), permitting all non-commercial use, distribution, and reproduction in any medium, provided the original work is properly cited. 


\title{
Risk Prevention Mechanisms in Asymmetrical Inter-Company Situations: An Exploratory Study in Smes in the BTP in Cameroon
}

\author{
Mecanismes de Prevention de Risques Dans les Realtions Asymetriques \\ Interentreprises : Etude Exploratoire Dans les Pme du Btp au Cameroun
}

\author{
Alain Marcel Manga Menyomo ${ }^{\alpha} \&$ Nicole Patricia Tjani ${ }^{\sigma}$
}

\begin{abstract}
Resume- S'il est vrai que les relations asymétriques interentreprises offrent beaucoup d'opportunités aux différents partenaires et à la PME en particulier, il n'en demeure pas moins qu'elles soient à l'origine d'un certain nombre de risques. Alors, comment les PME se prémunissent-elles des risques liés aux relations interentreprises ? Pour y répondre, nous avons adopté une méthodologie basée sur une approche qualitative axée sur des entretiens semi-directifs auprès de quatre (04) PME du BTP. Afin d'identifier et de classer les différents mécanismes de prévention de risques mobilisés par les PME. Les résultats obtenus relèvent que ces PME mobilisent à la fois les mécanismes contractuels et extracontractuels. Mais, privilégient plus les mécanismes extracontractuels ou relationnels dans la prévention des risques.
\end{abstract}

Motsclés: relation asymétrique interentreprises, mécanismes de prévention de risques, risques, BTP, PME.

Abstract- While it is true that asymmetrical inter-firm relations offer many opportunities to the various partners and to the SME in particular, they are nonetheless the source of a number of risks. So, how do SMEs protect themselves from the risks associated with inter-firm relations? To answer this question, we have adopted a methodology based on a qualitative approach based on semi-directive interviews with four (04) SMEs in the construction industry. In order to identify and classify the different risk prevention mechanisms mobilized by SMEs. The results obtained show that these SMEs mobilize both contractual and extra-contractual mechanisms. However, they give more priority to extracontractual or relational mechanisms in the prevention of risks. Keywords: asymmetrical inter-company relations, risk prevention mechanisms, risks, construction and public works, SMEs.

\section{INTRODUCTION}

( a stratégie des entreprises en situation de concurrence, d'après Alouat \& al. (2007), représente trois configurations: l'affrontement; l'évitement et la coopération. Cette dernière configuration connaît un intérêt tout à fait particulier. Elle

Author a: Ecole Normale Supérieure d'Enseignement Techniques (ENSET-Université de Douala) BP: 1872 Douala-Cameroun.

e-mail: mangaalain02@gmail.com

Author б: Institut des Beaux-Arts (IBA - Université de Douala) BP : 2701 Douala-Cameroun.e-mail: nicolepatriciat@yahoo.fr est, semble-t-il, liée à la mondialisation de l'économie, à l'internationalisation des marchés et à la turbulence technologique (Lee \& al., 2010; Hitt \& al., 2012; McGrath \& Kim, 2013; Simonin \& Gates, 2013). De plus, la coopération interentreprises connaît un développement fulgurant depuis plus de trois décennies, comme l'attestent de nombreuses études (Beamish \& Killing, 1997; Ouedraogo, 2003; Lecoq, 2003; Blanchot, 2006). Ces relations apparaissent incontournables dans la vie d'une entreprise. Pour plusieurs raisons, les entreprises évitent de plus en plus les comportements d'isolement et optent pour le regroupement. Ce qui les rend plus fortes et leur permet, entre autres, de réaliser des économies d'échelles (Garette \& Dussauge, 1995; Garcial-Canal \& al., 2002). D'ailleurs, c'est ce qui explique le fait que les entreprises multinationales soient à la quête de nouvelles sources d'avantages concurrentiels susceptibles de contribuer à l'amélioration de leur performance globale (Mayrhofer, 2007) tout en justifiant le développement effréné des rapprochements des entreprises, rapprochements qui visent d'une part, l'intérêt collectif (Kogut, 1988 ; Parkhe, 1993) inscrit dans une tendance compétitive afin d'accroître, protéger ou maintenir un avantage individuel (les Relations Interentreprises comme un vecteur privilégié du développement à l'international); et, d'autre part, une relation commune (Gulati, 1998 ) à la recherche des coentreprises formelles et des prises de participation (la solution même aux problèmes des entreprises lorsque celles-ci connaissent certaines difficultés liées au fonctionnement). En réalité, et comme l'affirme Cheriet (2009) aucune entreprise ne peut actuellement mener une stratégie « indépendante » des autres acteurs de l'environnement.

Le recours à une Relations Interentreprises implique non seulement un double centre de décision mais également sources de risques financiers et stratégiques (Montfort cité par Jaouen, 2006). Ce d'autant plus que ces relations sont des relations asymétriques c'est-à-dire nouées entre partenaires présentant des différences considérables : taille, de capacités financières, zone géographique, de mode de 
gestion (d'Iribarne \& al., 2002; Salk \& Shenkar, 2001). La PME, dans sa généralité, ne saurait échapper à cette réalité. Présentée comme le partenaire le plus vulnérable et par ricochet le plus exposé aux multiples risques inhérents à la relation (Sapienza \& Stork, 2001).

Face à ces difficultés, il devient urgent pour la PME de développer des mécanismes qui lui permettraient de se prémunir de ces risques. Ainsi déroulé, il est clair que la PME camerounaise en générale et celle du BTP en particulier n'échappe pas à cette réalité. Le secteur est en plein essor. D'ailleurs, depuis le début de l'année 2012, le Cameroun renvoie l'image d'un vaste chantier à la faveur des grands projets d'infrastructure qui y ont cours notamment dans le cadre du vaste programme des Grandes Réalisations du plan d'urgence trelations interentreprisesnnal lancé par le gouvernement en 2015 ; de la préparation du Championnat d'Afrique de football féminin, que le Cameroun a abrité entre novembre et décembre 2016; le CHAN de 2020 ; ou encore de la CAN de football masculin, que le pays accueillera en 2022 et qui était initialement prévue en 2019 mais qui a connu une reprogrammation faute de la réalisation dans les délais des infrastructures pouvant abriter un tel évènement. Tous ces éléments, nous confortent encore plus dans notre choix du secteur d'activité pour comprendre les origines et les motifs de ces insuffisances. Nous nous disions que cette étude nous permettra de mieux appréhender le fonctionnement des entreprises de ce secteur et mieux encore la place et le rôle que pouvaient jouer les PME locales dans l'exécution ou dans l'accompagnement de ces réalisations entre autres. Tant on relève que le problème sous-jacent est le plus souvent celui de l'incapacité technique et financière de ces prestataires à satisfaire aux exigences de leur commande alors que d'énormes opportunités d'affaires restent à saisir. II se trouve donc que la PME du Cameroun ne peut disposer de tous ces atouts. D'où ; il devient impératif pour cette dernière de nouer des relations avec des multinationales, mieux équipées et plus expérimentées. Ceci dans l'espoir d'acquérir entre autres un savoir-faire, des connaissances et des compétences spécifiques, avoir accès à la technologie. Les spécificités du secteur d'activité I'y obligeant. II s'avère dès lors indispensable pour ces PME, de mettre en place un ensemble de dispositifs leur permettant de se prémunir des multiples comportements non souhaités.

Dans le cadre de leurs travaux, Das \& Teng (2001) proposent trois types de contrôle: le contrôle basé sur des outils formels ; le contrôle comportemental et le contrôle social. Et dans le même ordre d'idée, Peillon (2001) propose deux types de mécanismes : des mécanismes contractuels ou transactionnels (contrat) et mécanismes extra-contractuels ou relationnels (confiance, engagement). Dans le cadre d'une étude sur les cas de collaborations technologiques entre PME sud-africaines et multinationales, Sawers \& al. (2008) soulignent que l'un des risques encourus par les PME dans leurs relations asymétriques est le risque de flux inattendus. Ces derniers proposent aux PME, pour répondre à ces risques, d'exiger des garanties formelles (contrat) et de développer des garanties informelles (confiance, engagement, partage d'informations ou communication). Partant de ce qui précède et dans le cadre de cette étude, nous allons nous atteler, à travers une étude exploratoire, de mettre en exergue les mécanismes mobilisés par les PME du BTP pour se prémunir des risques inhérents à la relation.

Ainsi présenté, il serait opportun de nous questionner sur la manière dont les PME procèdent pour se prémunir des risques inhérents à la relation. En d'autres termes comment les PME se prémunissentelles des risques liés aux relations interentreprises ? Cette question principale suscite des interrogations suivantes: quels sont les risques auxquels sont exposées les PME dans le cadre de ces relations asymétriques? Quels sont les différents mécanismes de prévention de risques mobilisés par les PME? Quelle peut être leur ordre de priorité ?

Répondre à cette question nous nous sommes fixé pour objectif principal de comprendre l'intégration des mécanismes de prévention des risques dans la gestion des Relations Interentreprises de répertorier. les risques auxquels sont exposés ces PME, d'identifier les différents mécanismes de prévention de risques mobilisés par ces PME et de les classifier.

\section{il. Management de la Relation} ASYMETRIQUE INTERENTREPRISES : LES Differents Mecanismes de Prevention

\section{DE Risques}

Les relations asymétriques demeurent relativement peu étudiées (Chtourou \& Laviolette, 2005) et spécifiquement difficiles à gérer. Elles sont identifiées par une multitudes de critères : la taille, la structure de gouvernance, l'origine géographique, la culture organisationnelle, l'expérience. dans la relation, la capacité d'apprentissage organisationnelle (Assens \& Cherbib, 2010 ; Perez \& al., 2012 ; Mohamadou, 2017).

\section{a) Risques inhérents aux relations interentreprises}

S'il est vrai que les relations asymétriques interentreprises offrent beaucoup d'opportunités aux différents partenaires et à la PME en particulier comme le souligne Puthod (1998) cité Cheriet (2016, p. 98) il n'en demeure pas moins qu'elles soient à l'origine d'un certain nombre de difficultés ou de risques; car l'asymétrie. observée entre les partenaires présente déjà une forte incitation à tricher à partir du moment où cette différence porte en elle-même les gènes de la complexité. C'est ce qui amène Dussauge \& Garette (1997) à souligner que l'établissement et surtout le 
fonctionnement d'une relation de coopération asymétrique rencontre des problèmes tout à fait particuliers provenant à la fois la cohabitation entre les centres de décisions multiples et de l'existence des conflits d'intérêt entre les partenaires. Dans le même ordre d'idées Montfort (1997) cité par Jaouen (2008) relève que le recours à une relation partenariale implique non seulement un double centre de décision, mais aussi est une source de risques financiers (coûts additionnels, Baisse de revenus) et stratégiques (perte d'autonomie, conflits de souveraineté, absorption du savoir-faire, position de marchandage défavorable, perte d'identité, de flexibilité stratégique ou de chance de nouer des accords avec d'autres partenaires). Dans la même logique, Blanchot (2006), recense trois types d'obstacles dans l'existence des relations partenariales: l'individualisme, l'incertitude et l'incompréhension. De même, d'Iribarne \& al. (2002), Salk \& Shenkar (2001) estiment que la relation de coopération est d'autant plus délicate qu'elle concerne les entreprises de pays différents et plus particulièrement entre entreprises du Nord et entreprises du Sud. L'asymétrie qui résulte. qui résulte de ce différentiel cause de multiples disparités tant en termes de culture et de compétences que de pouvoir (Sapienza \& Stork, 2001) et peut par conséquent appauvrir la communication et rendre l'apprentissage mutuel plus difficile entraînant ainsi incompréhension et méfiance. Ce qui pousse Delerue (2004) à qualifier le partenariat, de stratégie instable, risquée et dangereuse.

Ceci semble d'autant plus évident que, lorsque les partenaires présentent un déséquilibre en termes de taille et de culture, il y a inéluctablement un ensemble de comportements non souhaités et d'opportunismes. Le risque d'opportunisme constitue l'obstacle majeur auquel sont confrontés les partenaires dans les relations interentreprises. Dans la mesure où il incite l'aléa moral tout en poussant les partenaires à adopter des comportements de free-rider, à surexploiter une ressource commune, ou àtenter un hold-up de la quasi-rente issue de leur coopération (Peillon, 2001). D'ailleurs, Williamson (1985) en relève trois types d'opportunisme:

> «L'opportunisme sous forme de sélection adverse » qui est un opportunisme ex-ante basé sur la présence d'asymétrie... (Akerlof, 1970) ;

> «L'opportunisme sous forme de hold-up » c'est un opportunisme ex-post. II se caractérise par la modification des comportements qui ne reflètent plus ce qui avait été prévu par les partenaires (Klein \& al., 1978);

> «L'opportunisme sous forme de hasard moral »: c'est une forme de tricherie pendant la phase. pendant la phase d'exécution du contrat et rendu possible par l'incomplétude des contrats (Koenig, 1993).

En outre on observe un manque de confiance, une dépendance unilatérale et une domination par le plus grand $^{1}$.

Tout ceci contribue à fragiliser la relation interentreprises, par conséquent, constitue une menace à la stabilité, à la longévité, au succès et même à la performance de la dite relation.

Le tableau ci-dessous nous permet de ressortir quelques risques identifiés dans la littérature.

\footnotetext{
${ }^{1}$ Le niveau d'interdépendance fait référence au degré avec lequel chaque partenaire aura besoin de l'autre pour atteindre ses objectifs car plus l'interdépendance est élevée plus les partenaires trouvent un intérêt pour que la relation se prolonge et, par conséquent, s'impliquent d'avantage dans la relation, cherchent à éviter la rupture et à gérer au mieux cette relation. (Das \& Teng, 2003 ; Fréchet, 2002)
} 
Tableau 1: Synthèse des risques inhérents aux relations interentreprises

\begin{tabular}{|c|c|c|}
\hline Nature du risque & Risques associés & Auteurs \\
\hline $\begin{array}{l}\text { Caractéristiques } \\
\text { structurelles des partenaires }\end{array}$ & $\begin{array}{l}\text { Difficultés pour la PME de: } \\
\text { Identifier la personne adéquate à qui s'adresser ; } \\
\text { Avoir l'attention des décideurs; } \\
\text { Difficulté à concrétiser les idées développées }\end{array}$ & $\begin{array}{l}\text { Minshall \& al. (2010) } \\
\text { Prashantham \& Birkinshaw (2008) }\end{array}$ \\
\hline Risques relationnels & $\begin{array}{l}\text { Risques de dépendance; Risques de conflits ; } \\
\text { Risques d'incompréhension; } \\
\text { Risques de comportement opportuniste ; } \\
\text { Risques d'absorption ; Risques d'appropriation du } \\
\text { savoir-faire de la PME par la multinationale; } \\
\text { Risques d'abandon une fois que la PME n'a plus } \\
\text { rien à offrir ; Risques d'opportunisme. }\end{array}$ & $\begin{array}{c}\text { Mohamadou (2014) ; Barabel \& al } \\
\text { (2015) ; Fréchet (2007); } \\
\text { Kalaignanam \& al. (2006) ; } \\
\text { Delerue \& Simon (2005) ; } \\
\text { Delerue (2004) ; Alvarez \& Barney } \\
\text { (2001) ; Nooteboom \& al. (1997) ; } \\
\text { Das \& Teng (2001) }\end{array}$ \\
\hline $\begin{array}{c}\text { Risques liés aux objectifs } \\
\text { stratégiques des } \\
\text { partenaires }\end{array}$ & $\begin{array}{l}\text { Difficulté d'identification du bon interlocuteur } \\
\text { (identifier les personnes clés) }\end{array}$ & $\begin{array}{c}\text { Minshall \& al. (2010); } \\
\text { Prashantham \& Birkinshaw (2008) }\end{array}$ \\
\hline $\begin{array}{l}\text { Risques liés aux ressources } \\
\text { et capacités des partenaires }\end{array}$ & $\begin{array}{l}\text { Départ des meilleurs employés } \\
\text { Perte des compétences } \\
\text { Capacité financière } \\
\text { Disponibilité des équipements }\end{array}$ & Das \& Teng, (1999) \\
\hline
\end{tabular}

Source: Élaboré par l'auteur sur la base de la revue de la littérature.

Globalement, les recherches ont pu montrer que, malgré les avantages que peuvent tirer les PME des alliances asymétriques, elles courent aussi certains risques découlant des asymétries avec leurs partenaires. Et puisqu'elles semblent être les plus exposées aux risques, il paraît judicieux de s'y pencher. C'est pourquoi nous focalisons notre recherche sur la compréhension de l'intégration des mécanismes de prévention des risques dans la gestion des relations interentreprises afin d'aboutir à des solutions de relation gagnant-gagnant, comme le stipulent Seppänen \& al., (2007). Cette recherche porte essentiellement sur la PME.

L'enjeu majeur auquel le pilotage de la relation interentreprises doit répondre est ainsi d'éviter l'apparition des comportements opportunistes de la part des partenaires, de lutter contre l'incertitude, car ils sont nuisibles à l'efficacité de la coordination et sont sources d'importants coûts de transaction. II devient urgent pour la PME de développer des mécanismes qui lui permettraient de se prémunir de ces risques.

\section{b) Identification théoriques des mécanismes de prévention de risques}

Dans les relations asymétriques interentreprises, l'exercice d'anticipation constitue un aspect déterminant puisqu'il permet de se projeter sur les résultats probables, l'évolution, la nature de la relation entre partenaires, les éventuels risque, comment s'en prémunir et l'entité commune (Cheriet \& Dikmen, 2008). Ainsi, l'interface entre les partenaires doit être clarifiée en ce qui concerne les mécanismes de prévention à mettre en place dans les relations interentreprises.

Définis comme un ensemble de mesures prises pour empêcher la survenue d'un évènement non désiré, les mécanismes de prévention de risques constituent le dispositif à travers lequel les partenaires vérifient l'atteinte des objectifs, la protection des investissements et la conservation des informations essentielles pour l'opérationnalisation de la relation. D'après Killing (1983) cité par Yan (1998), les mécanismes de prévention relèvent des capacités des alliés à influencer les décisions stratégiques et à optimiser les choix opérationnels les plus importants. Et représentent pour Geringer \& Hebert (1989), un processus qui peut influencer les comportements et les résultats d'une entité en exerçant le pouvoir et l'autorité sur le déroulement des relations interentreprises. Ces mécanismes se matérialisent, dans les travaux de Yan \& Gray (1994), par la capacité à influencer les décisions opérationnelles, le management et la structure de gouvernance de relation. Vu sous cet angle, anticiper dans toute ou partie de la relation aiderait indiscutablement les partenaires en général et la PME en particulier à prévoir et à exposer moins leurs différentes stratégies, leurs atouts et forces technologiques ou d'autres actifs stratégiques à l'extérieur du groupe. Face au comportement opportuniste des multinationales, les PME peuvent se prémunir comme l'indiquent Das \& Teng (2001) à trois types de contrôle dans les relations asymétriques interentreprises:

- le contrôle (ou outputs) basé sur des outils formels comme le contrat;

- le contrôle comportemental (ou processus) qui a pour objet de vérifier le fonctionnement de la relation à travers des procédures et un partage de l'information;

- le contrôle social (ou informel) qui vise à créer une culture commune, des valeurs, des principes et des normes partagées. 
Dans le même ordre d'idée, Alvarez \& Barney (2001), en s'appuyant sur ce que Sawers \& al. (2008) ont convenu de retenir les garanties formelles en faisant allusion au contrat et des garanties informelles parlant de la confiance pour faire allusion aux mécanismes de prévention de risques. II est évident que les PME, pour s'en sortir, devraient mobiliser les mécanismes formels et informels.

De manière générale, la littérature distingue deux types de mécanismes de prévention: les mécanismes transactionnels ou formels et les mécanismes relationnels ou informels. Peillon (2001) se situe quasiment dans la même logique. Et préconise la prise en compte des mécanismes extra-contractuels (relationnels) et des mécanismes contractuels (transactionnels).

Face au comportement opportuniste des multinationales et comme le stipulent Lambe \& al. (2002) dans la théorie de l'échange sociale, les relations interentreprises sont essentiellement motivées par la recherche de l'intérêt individuel et par la perspective de ce qu'elles peuvent générer, c'est-à-dire les retombés escomptés. Cette théorie issue, comme le soulignent Moorman \& Byrne (2005), des travaux anthropologiques de Mauss (1923-1924) et, transposée aux sociétés contemporaines, précisent les bases motivationnelles des attitudes et comportements des employés. Cet échange social d'après Blau (1964), implique lui-même une réciprocité reposant sur trois principes: l'investissement, la confiance et l'engagement. Mais, quoique les parties exercent leur devoir de réciprocité, la forme de l'échange varie suivant les partenaires générant divers comportements et attitudes.

Et puisque les partenaires doivent survivre et faire évoluer la relation de coopération sans oublier que les alliés peuvent les désarmer. Les partenaires négocient le design de la structure de gouvernance et les mécanismes de prévention afin de réduire le risque de comportement opportuniste. L'interface entre les partenaires doit être clarifiée en ce qui concerne les mécanismes de prévention à mettre en place dans les relations interentreprises. Les partenaires vérifient, à travers les mécanismes de prévention, l'atteinte des objectifs, la protection des investissements et la conservation des informations essentielles pour l'opérationnalisation de la relation.

Pour s'en sortir, la PME devrait mobiliser les mécanismes lui permettant de se prémunir des éventuels risques inhérents à la relation. Dans la littérature, plusieurs mécanismes sont identifiés c'est ainsi que Das \& Teng (2001) distinguent trois types de contrôle dans les relations asymétriques interentreprises:

- le contrôle (ou outputs) basé sur des outils formels comme le contrat;

- le contrôle comportemental (ou processus) qui a pour objet de vérifier le fonctionnement de la relation à travers des procédures et un partage de l'information;

- le contrôle social (ou informel) qui vise à créer une culture commune, des valeurs, des principes et des normes partagées.

Cette revue de la littérature sur les mécanismes de prévention de risques nous permet de formuler la Proposition suivante :

P1: les mécanismes de prévention de risques contribuent considérablement à la prévention de risques inhérents à la relation interentreprises

\section{Les mécanismes contractuels}

Pour l'approche contractuelle, la coordination repose sur les contrats. Dans cette optique, la coordination des relations interentreprises est fondamentalement basée sur des mécanismes contractuels. Les approches contractualistes sont caractérisées par un " consensus minimal » sur la nature et le rôle des contrats (Broussoau, 1989). Elles envisagent le pilotage de la relation interentreprises du point de vue contractuel, c'est-à-dire se fondant exclusivement sur des mécanismes contractuels pour lutter contre les éventuels comportements opportunistes que pourraient adopter les partenaires. La nécessité des mécanismes contractuels, du fait de l'imperfection de l'environnement informationnel, comme le souligne Peillon (2001) n'est plus à démontrer. Cette imperfection informationnelle rend donc les contrats indispensables parce qu'elle est à l'origine des comportements opportunistes. Parmi ces approches contractuelles, nous distinguons: les théories la TCT, la théorie de l'agence, la théorie des contrats, et la théorie institutionnelle.

L'hypothèse selon laquelle le contrat constituerait l'unique solution aux opportunismes nous semble dès lors contestable. II nous serait important de chercher d'autres formes de prévention autres que le contrat et qui soient efficace. D'où, la nécessité de compléter les mécanismes contractuels. Car ceux-ci présentent des limites dues, d'une part, à la nature des comportements des agents économiques et d'autre part, aux caractéristiques des contrats (incomplétude, efficacité relative, complexité, coûts élevé, inadaptation). Pour ce qui est de l'incomplétude par exemple, la rédaction du contrat implique que les parties déterminent précisément les performances à atteindre par les parties. Or, du fait de l'incertitude sur l'avenir, de la complexité des décisions à prendre, de la rationalité limité, il serait impossible de rédiger un contrat complet. Ce qui nous amène à considérer que les contrats ne peuvent être optimaux comme le stipule la théorie des contrats. Et nous pouvons penser qu'il existe des mécanismes différents qui peuvent eux aussi permettre de limiter les risques. D'ailleurs, les mécanismes transactionnels ou contractuels qui induisent les contrats tendent, selon Schann (1988), à être 
prévisibles, réguliers et impliquent des transferts explicites d'informations.

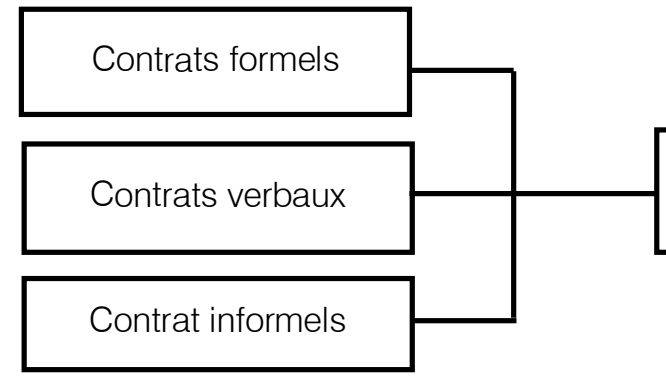

Mecanismes Contractuels

Source: Élaborée par l'auteur

Figure 1: Mécanismes de prévention contractuels

C'est pourquoi, dans l'optique de compléter les contrats qui restent incomplets, il serait utile de mettre en œuvre d'autres dispositifs pour gérer l'opportunisme des partenaires. Ainsi, d'autres moyens ont été identifiés dans la littérature. Dès lors, le pilotage des Relations Interentreprises ne pourrait plus seulement être contractuel (transactionnel), mais d'avantage extracontractuel (relationnel). II s'agit des mécanismes extracontractuels.

\section{$>$ Les mécanismes extra-contractuels}

L'analyse économique traditionnelle a toujours considéré l'individu comme un homo-œconomicus et par conséquent, l'individu est entièrement tourné vers la recherche de son intérêt, c'est égoïste pur. Les relations individuelles sont donc envisagées en termes de conflits. Mais depuis quelques années, l'impact des relations sociales sur l'action économique a commencé à être pris en considération notamment, le fait que les relations interpersonnelles ne soient plus exclusivement basées sur la recherche de l'intérêt personnel dans les échanges hors marché tel que les échanges de savoirfaire, de connaissances (Peillon, 2001, p. 81).

Le contrat seul n'est donc plus suffisant pour la prévention des risques (Ménard, 1994b). En effet, d'une part les contrats ont des limites, et, d'autre part, dès lors que l'on prend en compte le caractère temporel et dynamique de la coopération interentreprises, on est conduit à envisager des mécanismes de prévention pouvant être construits par les partenaires. D'autres facteurs tels que les normes sociales, les relations personnelles, la confiance, loyauté, réputation.

- La confiance un frein aux risques des relations interentreprises

Les organisations cherchent à faire face aux risques en mettant en place des mécanismes qui vont leur permettre d'orienter leurs réponses à l'imprévu (Lazaric \& Lorenz, 1998, cité par Peillon, 2001, p. 91). La confiance est alors nécessaire pour faire face aux inévitables contingences imprévues. Elle renvoie à la volonté d'une partie à s'appuyer sur les actions d'une autre partie dans une situation comportant des risques et des incertitudes. Elle correspond à une situation dans laquelle une partie croit en la fiabilité et l'intégrité de l'autre partie (Morgan \& Hunt, 1994). Ainsi, elle facilite la compréhension mutuelle et permet un bénéfice mutuel. Et comme le précisent Koenig \& Van Wijk (1992), de la confiance apparaît un mode de contrôle informel qui gouverne des acteurs mutuellement identifiés. Par conséquent, elle permet de réduire l'incertitude relative au comportement mutuel à l'aide d'un processus d'autocontrôle. D'une manière générale, nous pouvons, dans la suite de Lee \& Cavusgil (2006), insinuer qu'elle accélère le transfert des connaissances en facilitant l'interaction entre les parties impliquées, ce qui leur permet de localiser les informations clés.

En outre, la confiance est sensée augmenter le niveau d'engagement car les relations basées sur la confiance sont jugées de manière positive. Elle inscrit les relations interentreprises dans une approche relationnelle. En faisant nôtre la définition de Koenig \& Van Wijk cité par Ouedraougo (2003), la confiance sera prise ici comme un processus qui met en œuvre des anticipations et des obligations entre deux parties. Et en tant que telle, elle sera comparable à un dispositif de prévention des risques dans les relations interentreprises. Elle est considérée suffisamment complexe car elle implique aussi bien des dimensions individuelles, que des dimensions interpersonnelles, sociales, politiques et économiques (Gambetta, 1988). La confiance rend cette situation gérable dans la mesure où elle réduit le risque de tromperie ou de comportement opportuniste. Pour cela, la confiance présuppose une prise de décision dans une situation de risque, où le risque est attribuable à la possibilité de comportement opportuniste des autres. Mohamadou (2017), affirme que malgré les asymétries et contrairement à bon nombre d'auteurs, la confiance ne peut se développer entre les partenaires que si et seulement si des dispositions sont prises par les parties pour favoriser la relation. En tout état de cause, la confiance apparait comme un mécanisme permettant de réduire l'incertitude comportementale. Ainsi, la confiance présuppose une prise de décision dans une 
possibilité que les autres se comportent de façon opportuniste.

- La communication facteur de cohésion dans les relations interentreprises

La communication est définie par Brulhart (2002) comme le partage formel et informel d'informations pertinentes et actualisées entre les entreprises partenaires. Sa qualité constitue une des clés du succès dans la mise en place d'un projet coopératif. Une bonne communication constitue un facteur de cohésion entre partenaires. Un fort degré de communication aura une influence positive sur le degré de confiance entre les partenaires et les managers chargés de gérer la relation. Dans toute relation bilatérale notamment dans un contexte de relations interentreprises, quand deux partenaires désirent communiquer entre eux, ils cherchent, entre autres, à échanger des informations, des idées et des connaissances ou des attitudes en commun. Elle permet comme l'affirment Anderson \& Weitz (1992), de prévenir les éventuels malentendus et de réduire les risques de conflits et de dysfonctionnement entre les différents acteurs. Le partage d'informations contribue à réduire la distance et les différences entre les parties. Principal instrument de traitement des conflits, la communication semble être l'élément indispensable du bon fonctionnement de la relation. Elle traduit, en tant que clé de collaboration, un facteur d'émergence de la confiance. Car une bonne communication favorise des contacts fréquents, amène les parties à s'informer à l'avance des changements qui pourraient avoir une incidence sur la relation, facilite une ouverture réciproque (Cao \& al., 2011).

\section{- L'engagement}

II peut être décrit comme la volonté des partenaires de relations interentreprises. de faire des efforts au nom de la relation en réduisant la menace de comportement opportunistes (Mohr \& Spekman, 1994). A ce titre, il réduit les coûts de transaction et les coûts associés au partenariat. II s'entend comme une dévotion d'un partenaire à la coentreprise (Christoffersen, 2013). Pour Inkpen (2000), le degré d'engagement entre partenaires dans les RIE, requiert deux aspects: le degré d'ouverture des partenaires degré de communication, culturel, linguistique...) et l'intensité de leurs relations (volonté, effort mutuel). Par conséquent, l'engagement peut contribuer à atténuer le comportement opportuniste des partenaires. $\mathrm{Ce}$ d'autant plus que, les partenaires souhaitent renforcer cette relation et surtout s'investissent pour la rendre pérenne (Groonroos, 1990). Vue sous cet angle, l'engagement favoriserait sans doute un comportement coopératif et créerait un sentiment d'unité induisant par la même occasion plus de confiance. Morgan \& Hunt (1994), l'assimilent à la croyance d'un partenaire que la relation en cours est si importante qu'il faut engager le maximum d'efforts pour la maintenir. II est cependant nécessaire de signaler que le degré d'engagement reste étroitement lié au degré de confiance.

- La réputation facteur d'anticipation aux comportements opportunistes

Elle est fondée sur la notion de confiance calculée. Elle provient de l'interaction des agents recherchant leur intérêt personnelle. Les parties restent dans la relation tant qu'elles considèrent que c'est dans leur intérêt de le faire.

La réputation n'est efficace que si sa diffusion est assurée et donc si la communication entre les acteurs est suffisamment développée. On voit ici poindre l'importance de la proximité entre les acteurs. Au sein des coopérations interentreprises, la réputation fonctionnera d'autant mieux que les partenaires sont proches géographiquement. Ce point est illustré par le modèle du district industriel (Beccatini, 1990), dans lequel les firmes se connaissent et se reconnaissent, la proximité géographique permettant à la fois la répétition des engagements, leur vérification quotidienne et la diffusion de la variable réputation.

Au total, la réputation peut constituer, pour les partenaires de la coopération, un mécanisme permettant de faire respecter les contrats et un frein à l'opportunisme ex post. De plus, elle permet de réduire les coûts de rédaction du contrat de coopération et d'éviter les coûts liés à la mise en œuvre des mécanismes permettant d'assurer le respect de ce contrat par les partenaires. Au sein des coopérations interentreprises, la réputation représente donc un mécanisme complémentaire au contrat.

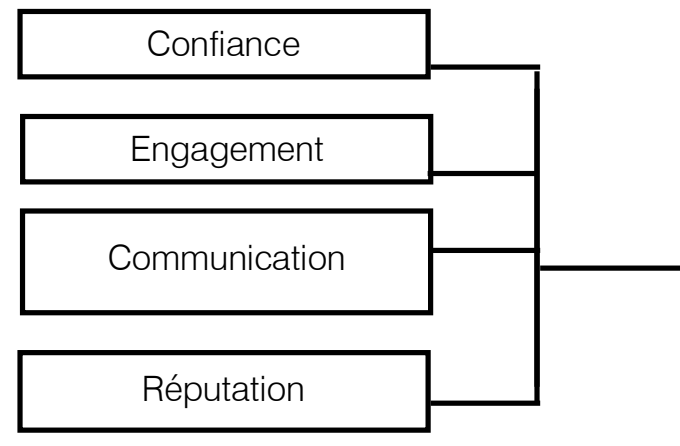

Mecanismes Extra-Contractuels

Figure 2: Mécanismes de prévention extra-contractuels 
P.2: les mécanismes contractuels sont prioritaires aux mécanismes extra-contractue/s

Notre revue de la littérature met en évidence la théorie du contrat avec la généralisation des contrats dans toutes les relations. Toutefois, elle s'avère insuffisante pour expliquer les relations interentreprises. $\mathrm{Ce}$ qui nous conduit à opter pour une démarche alternative en mobilisant plusieurs théories évolutionistes. Au-delà de l'explication des relations interentreprises, la littérature nous a permis de mentionner les mécanismes de pilotage de ces relations et ceux qui ont été identifiés ont pu être regroupés en deux grands ensembles. Qui nous ont servi de fil conducteur pour la suite de ce travail.

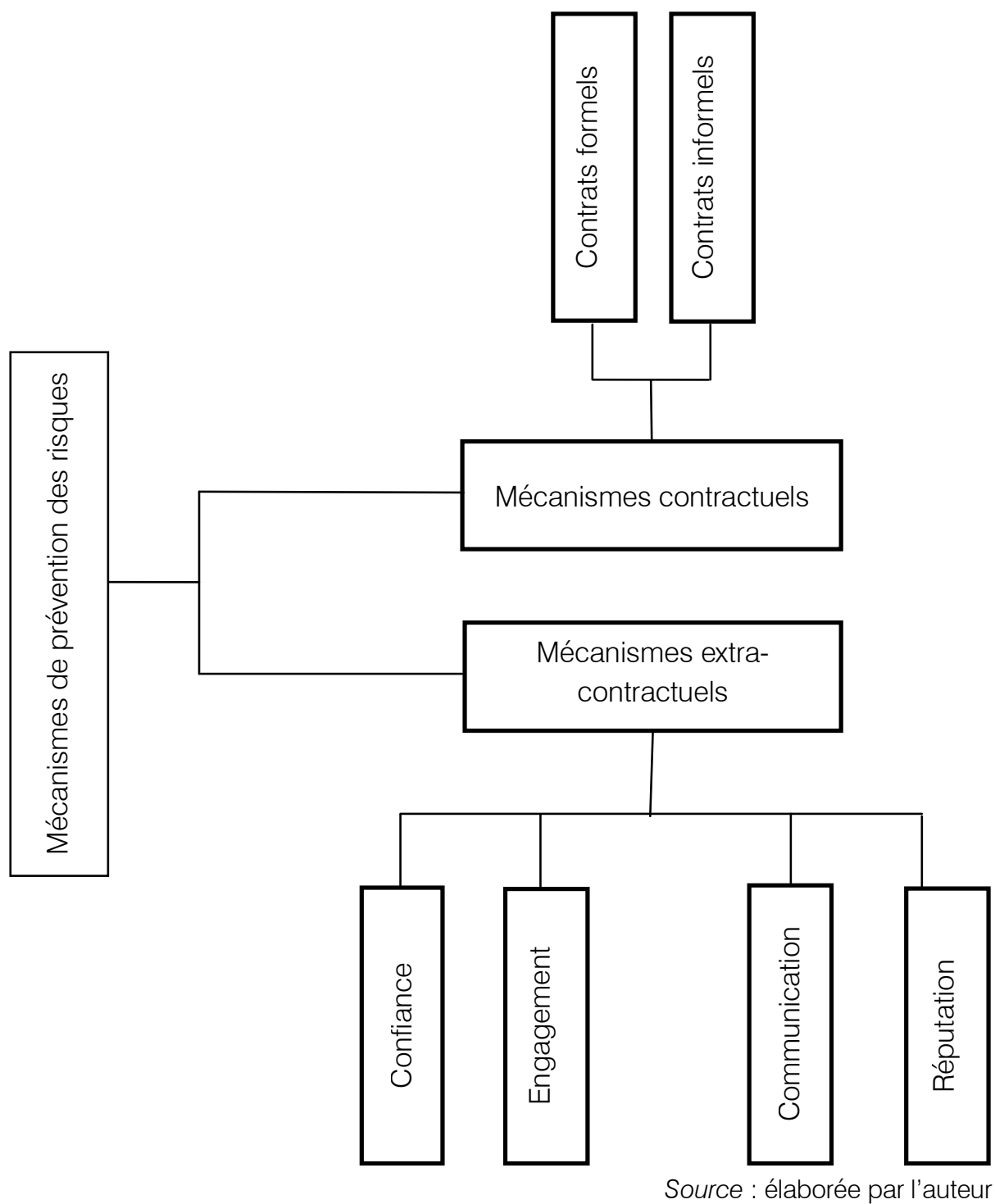

Figure 3: Mécanismes de prévention des risques

\section{ili. Méthodologie de la Recherche}

Afin de mener à bien cette étude, nous avons inscrit notre démarche dans le paradigme interprétativiste. Cependant, nous n'avons pas exclusivement adopté un raisonnement inductif comme le préconise ce paradigme mais nous avons opté pour une démarche hybride qui consiste à faire des allers retours entre la théorie et la démarche sur le terrain :la démarche abductive. L'approche exploratoire utilisée repose sur l'étude de cas.
Nous avons orienté nos interviews vers des acteurs impliqués dans la mobilisation des mécanismes de prévention des risques des PME en relation interentreprises du BTP. La méthode qualitative nous semble favoriser un contact direct avec les acteurs du terrain et paraît de cette manière à même de fournir, d'après Miles \& huberman (2003) cités par Abitbol (2012, p.105) «des explications riches et solidement fondées de processus ancrés dans un contexte local ». Ce point de vue que nous partageons, nous laisse 
penser que la méthode qualitative est ici la mieux adaptée. À terme, il s'agit de procéder avec efficacité à une identification et à une classification des mécanismes de prévention de risques mobilisés par les PME du le BTP. Cette étude est faite auprès de quatre (4) PME, taille suffisante pour conduire selon Eisenhardt (1989), une étude qualitative de qualité. II s'agit des PME engagées dans une relation. Quatre critères ont guidé notre choix: les PME en relation avec au moins une multinationale; la disponibilité de la PME et l'accessibilité à l'information recherchée; la spécificité du secteur d'activité dont l'importance n'est plus à démontrer au vu de l'intérêt que lui accordent les praticiens et les pouvoirs publics, enfin sur le thème pour mieux identifier les mécanismes de prévention de risques et appréhender leur classement

Pour la collecte des données, nous avons réalisé un guide d'entretien type. Ce choix nous a permis de comprendre et d'analyser le point de vue des acteurs retenus. Et les personnes interrogées sont: le propriétaire dirigeant(PDG), les directeurs techniques (DIRTECH), le Chef Projet (CHEFPRO), le directeur d'étude (DIRETU) et le directeur administratif (DIRADM) selon les quatre cas retenus.

Pour le traitement et l'analyse des données, plusieurs outils sont mobilisés : l'analyse des entretiens, les fiches de synthèses des entretiens et les codes que nous complétons par une analyse des fréquences et une étude de similitude/dissimilitude.

Le logiciel MAXQDA 2018 a été utilisé pour explorer les relations entre les codes. Cette démarche est axée sur la perception que les responsables des PME de ce secteur ont de les mécanismes de prévention et surtout sur leur hiérarchisation dans leurs relations avec les multinationales afin de prévenir les risques. Le tableau ci-dessous permet de résumer toute notre démarche.

\section{Tableau 2: Synthèse des choix méthodologiques}

\begin{tabular}{|c|c|c|}
\hline Choix & Option retenue & Déterminants \\
\hline Épistémologie & Interprétativiste & $\begin{array}{l}\text { Les frontières entre le phénomène étudié et le contexte } \\
\text { ne sont pas clairement identifiables, La vérité est } \\
\text { difficilement accessible, représentation de cette dernière }\end{array}$ \\
\hline $\begin{array}{l}\text { Type de } \\
\text { méthodologie }\end{array}$ & Qualitative & $\begin{array}{l}\text { Faiblesse des travaux théoriques, statut épistémologique } \\
\text { de l'identification et de la classification des mécanismes } \\
\text { de prévention de risques }\end{array}$ \\
\hline $\begin{array}{l}\text { Type de } \\
\text { raisonnement }\end{array}$ & Abductif & $\begin{array}{l}\text { démarche hybride qui consiste à faire des allers-retours } \\
\text { entre la théorie et la démarche sur le terrain }\end{array}$ \\
\hline $\begin{array}{lr}\text { Technique } & \text { de } \\
\text { recueil des données }\end{array}$ & $\begin{array}{l}\text { Études documentaires, entretiens semi- } \\
\text { directifs et observation }\end{array}$ & $\begin{array}{l}\text { Accès au vécu des acteurs, laisser émerger de nouveaux } \\
\text { concepts absents de la littérature, développer un niveau } \\
\text { de confiance avec les acteurs interviewés }\end{array}$ \\
\hline Niveau d'analyse & $\begin{array}{l}\text { Dimension } \\
\text { organisationnelle, et Stratégique de la } \\
\text { contribution des mécanismes de } \\
\text { prévention des risques dans la prévention } \\
\text { ou de la réduction des risques }\end{array}$ & $\begin{array}{l}\text { La caractéristique «anticipationnelle " des mécanismes } \\
\text { relationnels de prévention des risques comme facteurs } \\
\text { contribuant à la performance de la relation }\end{array}$ \\
\hline $\begin{array}{l}\text { Terrain } \\
\text { Recherche }\end{array}$ & PME du BTP & $\begin{array}{l}\text { PME du secteur BTP en relation avec une ou plusieurs } \\
\text { entreprises multinationales }\end{array}$ \\
\hline
\end{tabular}

Source: Synthèse de la littérature et la démarche empirique

\section{Resultats}

Après avoir présenté les caractéristiques des PME du BTP nous allons, dans un premier temps procéder à l'identification des mécanismes de prévention de risques mobilisés par les PME et le deuxième point nous permettra de procéder à la classification des mécanismes en fonction du poids que représentent chacun dans sa prise en compte par la PME.

\section{a) Caractéristiques des PME objet d'étude}

Toutes les entreprises de notre échantillon ont une espérance de vie d'au moins 6 ans, gage d'expérience. Ceci renvoie à la maîtrise des rouages et de la complexité du secteur. Elles font toutes parties du même secteur d'activité et couvrent tous les domaines (du gros cuuvre aux travaux de finition passant par l'expertise et les études et le conseil) liés à cette activité. La moyenne de l'effectif est de 36 employés permanents quoique faisant toutes recours à une main d'œuvre temporaire lorsque cela s'avère nécessaire. 
Tableau 3: Caractéristiques des entreprises

\begin{tabular}{|c|c|c|c|c|}
\hline Entreprises & Date création & Effectif & $\begin{array}{c}\text { Siège } \\
\text { social }\end{array}$ & Activités spécifiques \\
\hline Cas 1 & 1998 & 45 & Douala & Construction \\
\hline Cas 2 & 2014 & 22 & Yaoundé & Terrassement, décapage et pose de buse \\
\hline Cas 3 & 2009 & 40 & Yaoundé & $\begin{array}{r}\text { Etudes-contrôle et audit, études d'impact } \\
\text { environnemental, habillages muraux et décoration } \\
\text { intérieure travaux routiers, finitions }\end{array}$ \\
\hline Cas 4 & 2000 & 37 & Douala & $\begin{array}{r}\text { Construction des bâtiments, fabrication du } \\
\text { mobilier professionnel et domestique, fourniture } \\
\text { de béton, pose de caniveaux et aménagement et } \\
\text { voirie urbaine, voiries urbaines }\end{array}$ \\
\hline
\end{tabular}

Source: Élaboré par l'auteur

Les multiples risques auxquels sont exposées les PME, les poussent à déployer des mécanismes de protection. II ressort que ces entreprises, pour se prémunir des risques inhérents, mettent en place différents types de mécanismes.

\section{b) Mécanismes mobilisés par les PME}

Le contrat apparaît comme le mécanisme de prévention de risques incontournable pour toutes les PME du secteur BTP en relation interentreprises. Cela se trouve étayé par les répondants à travers les déclarations suivantes:

Le dispositif le plus évident est le contrat qui représente l'engagement entre les deux parties c'està-dire la multinationale et la PME celui-ci survient parfois au début de la relation ou parfois même à la fin et est le plus souvent écrit. Mais il reste vrai que certains accords sont verbaux ou se font au gré à gré sans fondements juridiques c'est-à-dire sur l'entente verbale des deux parties (Cas1_CHEFPRO)

Pour ce répondant, le contrat est incontournable dans une relation peu importe le moment où il survient mais il reste un dispositif indispensable.

Pour moi, le contrat c'est la paperasse on ne s'en sert que si on va en justice le vrai contrat c'est la parole d'un homme donné à un autre vraiment pour l'heure je ne sais pas si je peux changer d'avis. En réalité c'est la crédibilité, la loyauté, la confiance qu'on a l'un pour l'autre car il est important de garder son honneur et d'assumer ses engagements sans avoir besoin à tout prix que cela soit consigné sur $d u$ papier néanmoins le contrat écrit n'est qu'un élément de preuve au cas où? Et c'est d'ailleurs pourquoi en ce qui me concerne cette paperasse dans certains accords est souvent signée au milieu de l'exécution du marché voire même à la fin pour laisser des traces et surtout pour entrer en possession de son dû. En fait, ça c'est ma façon de voir les choses avec l'expérience que j'ai acquise sur les multiples chantiers et qui a contribué à développer mon intuition. D'ailleurs, c'est cette intuition qui me conduit à flairer les mauvaises affaires et à anticiper sur certaines choses. Je dois vous le dire, mon intuition n'est pas une simple intuition car, elle part du cumul $d^{\prime}$ expérience et elle est basée sur ma solide culture de gestion et c'est pourquoi je la qualifie moi-même, très souvent d'Intuition « rationnalisée » (Cas1_PDG)

De façon générale, le contrat est existant pour ce Cas1 mais il revêt un caractère ou un poids moins important que les autres types de mécanismes. D'ailleurs, ces deux répondants nous permettent de distinguer deux types de dispositifs: les dispositifs contractuels et les dispositifs non contractuels. II est donc identifié ici le contrat, les accords verbaux ; le gréà-gré, la crédibilité, la loyauté, la confiance, d'Intuition « rationnalisée ».

En dehors du contrat, qui ne garantit que le droit du plus fort c'est-à-dire de la multinationale, nous comptons sur les relations de confiance qui peuvent exister entre nous et qui sont plus susceptibles de nous garantir plus de visibilité, de partage d'information, la communication permanente et dans une certaine mesure l'engagement outre ces éléments, nous ne faisons absolument rien d'autre que compter sur la bonne foi de notre partenaire. Nous disons que quelque part dans le milieu des BTP on croit en Dieu et puis quand on croit on vit avec sa foi et advienne que pourra. Tout ce qu'on peut rencontrer on verra comment on peut contourner; rien n'est impossible au niveau des hommes. Je m'attache également les services d'un auditeur externe qui me rassure de la qualité de mes prestations (Cas2_PDG) 
Ce répondant identifie le contrat et la confiance, la communication et dans une moindre mesure l'engagement comme principaux mécanismes de prévention de risques.

Pour éviter que ces multinationales ne nous phagocytent, on s'appuie sur le contrat qui nous garantit un minimum de sécurité. Hors mis le contrat, II faut créer au maximum un climat de confiance, de convivialité, un climat d'amitié vous mettant à l'abri d'un certain nombre de risques. Parce que je me dis quand vous travaillez avec une multinationale dans tout ce que vous faites la relation humaine doit être privilégiée car s'il n'y a pas de relation humaine on sera toujours en train de courir derrière les risques (Cas3_PDG)

Tout comme dans les cas précédents, il ressort ici que les mécanismes mobilisés par cette PME et identifiés par ce répondant sont le contrat, la confiance.

Le respect de l'Ethique des affaires constitue, à mon avis, un mécanisme permettant d'anticiper sur les éventuels risques puisqu'impliquant un respect mutuel en affaire par conséquent des comportements responsables (Cas3_DIRETU).

Dans ce Cas3, ce répondant, en plus du contrat et de la confiance déjà mentionné par son collaborateur plus haut, relève qu'il faille également compter sur l'Ethique des affaires pour anticiper ou réduire les risques de la relation interentreprises.

Le contrat est en réalité notre principal élément de prévention. Tout est établi dans le contrat. II y a des pénalités pour empêcher les comportements déviants et obliger les uns et les autres à respecter leur engagement. C'est vrai qu'à côté du contrat, aussi exigent et contraignant qu'il soit, on tient compte des relations de personnes à personnes c'est-à-dire la crédibilité du responsable de l'entreprises. On peut également parler de la confiance qui, pour ma part, semble même être l'élément primordial pour le bon déroulement de la relation puisque la confiance m'amène à être sûr que mon partenaire même sans mon contrôle va exécuter, dans le sens de nos intérêts communs, le travail. La confiance, l'engagement et la loyauté peuvent aussi constituer un facteur important (Cas4_PDG)

Le contrat, la confiance, l'engagement, la loyauté et la crédibilité constituent pour ce répondant les mécanismes auxquels fait recours sa PME pour empêcher la survenue des risques.

Le contrat est exigible par la règlementation ce qui permet de légaliser la relation et en cas de problème d'avoir une voie de recours institutionnelle. II arrive très souvent que ce contrat soit boycotté par la multinationale. C'est pourquoi, il est nécessaire qu'en plus du contrat, qu'on compte sur la confiance et l'engagement entre les partenaires (Cas4__DIRADM).

Confiance, engagement et contrat se trouve être identifiés ici comme mécanismes de prévention de risques ce qui correspond aux dispositifs répertoriés par le PDG de la même PME.

Tout ce qui précède nous amène à tirer la conclusion suivant laquelle plusieurs mécanismes sont mobilisés par les Cas de notre étude pouvant être regroupés en deux catégories: les mécanismes contractuels et les mécanismes extra-contractuels.

Pour ce qui est des mécanismes contractuels on peut distinguer des contrats formels caractérisés par des contrats écrits et ayant un fondement juridique et des contrats informels basés sur les accords verbaux, des ententes, le gré à gré. Concernant les mécanismes extra-contractuels,: - la confiance, - l'engagement, - la communication ou le partage d'information globalement relevés par les répondants et déjà identifiés dans les travaux antérieurs (Peillon, 2001; Cherbib, 2014; Cheriet, 2008 ; Mohamadou, 2014) sont des dispositifs retenus par ces PME.

II ressort cependant qu'à côté de ces mécanismes, l'on a pu répertorier

- I'Intuition « Rationnalisée »

- l'Ethique des affaires. comme des mécanismes, au vu de la littérature parcourue, pratiquement non signalés et que nous pourrions classer dans la catégorie des mécanismes extra contractuels.

- Ces mécanismes, constituent pour les cas qui les utilisent, de précieuses armes pour prévenir tout comportements non désirés semble-t-il.

II faudra noter que l'intérêt de tous ces mécanismes est de contribuer ou de participer à la réduction du risque dans les relations interentreprises et de favoriser implicitement un meilleur pilotage de la relation et par ricochet la réalisation de la performance de la relation.

Ces mécanismes seront recensés dans le tableau ci-après pour repérer les mécanismes utilisés dans ce secteur d'activité et surtout comparer en fonction des Cas les mécanismes les plus pertinents: 
Tableau 4: Comparaison des mécanismes de prévention de risques

\begin{tabular}{|c|c|c|c|}
\hline & \multicolumn{2}{|c|}{ Mecanismes Contractuels } & \multirow{2}{*}{ Mecanismes Extra-Contractuels } \\
\hline & Contrat formel & Contrat informel & \\
\hline Cas 1 & \multirow{4}{*}{ 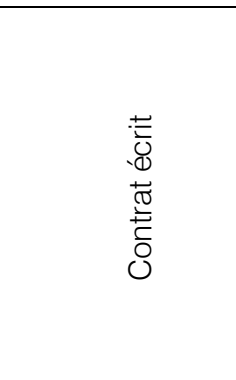 } & $\begin{array}{c}\text { Contrat verbal } \\
\text { Entente entre parties }\end{array}$ & $\begin{array}{c}\text { Confiance } \\
\text { Intuition « rationnalisée »; } \\
\text { Engagement ; communication }\end{array}$ \\
\hline Cas 2 & & $\begin{array}{c}\text { Entente entre parties } \\
\text { Négociations sous la table }\end{array}$ & $\begin{array}{c}\text { Confiance ; Communication ; } \\
\text { Engagement }\end{array}$ \\
\hline Cas 3 & & Contrat verbal & $\begin{array}{c}\text { Engagement ; Confiance } \\
\text { Ethique des « affaires » } \\
\text { communication }\end{array}$ \\
\hline Cas 4 & & $\begin{array}{l}\text { Négociation dans les couloirs } \\
\text { contrats verbaux }\end{array}$ & Confiance ; communication \\
\hline
\end{tabular}

Source: élaboré par l'auteur

c) Classification des mécanismes de prévention de risques

Les mécanismes de prévention de risques identifiés, il serait judicieux et indiqué de procéder à la classification de ces mécanismes en fonction du poids qu'ils représentent pour les PME objet de notre étude.
Il ressort que les mécanismes les plus sollicités par les Cas étudiés, si l'on procède au classement par catégories sont les mécanismes extra-contractuels soient $62,50 \%$ contre seulement $37,50 \%$ pour les mécanismes contractuels. Le tableau ci-dessous donne une illustration de cette réalité en fonction du poids de ces mécanismes extra-contractuels

Tableau 5: Mécanismes de prévention de risques privilégiés par les PME

\begin{tabular}{|c|c|c|}
\hline NATURE DU MECANISME & Fréquence & \% pourcentage \\
\hline MECA/EXTRA_CONTRACT (Relationnels) & 5 & 62,50 \\
\hline MECA/CONTRACT (Transactionnel) & 4 & 37,50 \\
\hline Totale & 8 & 100,00 \\
\hline
\end{tabular}

Source: élaboré par l'auteur

Parmi les mécanismes extra-contractuels, la priorité est donnée à la confiance avec un poids de $31,63 \%$, suivi de l'intuition «Rationalisée », de l'Ethique des affaires, de l'engagement et de la communication avec respectivement 22,45\%, $19,38 \%, 16,32 \%$ et $11,22 \%$ de poids voir Tableau 6 ci-dessous

Tableau 6: Mécanismes Extra-contractuels

\begin{tabular}{|c|c|c|}
\hline MECANISMES EXTRA-CONTRACTUELS & Fréquence & \% pourcentage \\
\hline Confiance & 31 & 31,63 \\
\hline Intuition « rationnalisee » & 22 & 22,45 \\
\hline Ethique des affaires & 19 & 19,38 \\
\hline Engagement & 16 & 16,32 \\
\hline Communication & 11 & 11,22 \\
\hline Totale & 98 & 100,00 \\
\hline
\end{tabular}

Source: élaboré par l'auteur

Le contrat formel, parce que exigible par la règlementation, constitue dans cette catégorie de mécanismes contractuel, le dispositif le plus postuler. Ceci en plus des contraintes légales peut se justifier par le fait qu'il confère aux partenaires des droits et la possibilité de recours en cas de non-respect des clauses. II représente $69,44 \%$ du poids des mécanismes contractuels contre seulement $30,56 \%$ pour les contrats informels qui à notre avis auraient pu être considérés comme des mécanismes extra-contractuels.

Tableau 7: Mécanismes contractuels

\begin{tabular}{|c|c|c|}
\hline MECANISMES CONTRACTUELS & Fréquence & \% pourcentage \\
\hline Contrat formel & 25 & 69,44 \\
\hline Contrat informel & 11 & 30,56 \\
\hline Totale & 36 & 100,00 \\
\hline
\end{tabular}

Source: élaboré par l'auteur 


\section{Discussion}

Le contrat apparait comme le seul mécanisme contractuel par excellence usiter par toutes ces PME. A côté de ce contrat, nous relevons que tous ces dirigeants ont recours et de manière informelle à des ententes et négociations moins conventionnelles.

Pour ce qui est des mécanismes extracontractuels, la confiance, l'engagement et la communication sont des modalités communes à tous ces cas. Le Cas1 et le Cas3 enrichissent cette liste de mécanismes extra-contractuels par:-|'Intuition « rationnalisée » et - l'Ethique des affaires.

Pour identifier les mécanismes nous nous sommes appuyé sur les travaux des auteurs qui nous ont précédés tels que: Geringer \& Hebert (1989), Mohr \& Spekman (1994), Peillon (2001), Das \& Teng (2001),), Sawers \& al. (2008), (Cheriet \& Dikmen, 2013), Cheriet (2016). Ces travaux nous ont conduit à retenir deux types de mécanismes : - les mécanismes contractuels ou transactionnels constitués du contrat formellement rédigé et du contrat informel matérialisé par les ententes verbales, le gré à gré; - les mécanismes extracontractuels ou relationnels illustrés par la confiance, l'intuition «rationnalisée », l'engagement, l'éthique des affaires, la communication ou partage d'information. D'ailleurs, si la confiance, l'engagement et la communication sont largement reconnus dans la littérature comme mécanismes, I'Intuition «Rationnalisée » et l'Ethique des affaires sont des mécanismes tout à fait nouveau et spécifique aux cas étudiés et mobilisés particulièrement et respectivement par les Cas 1 et Cas 3. Mais, de ces deux types de mécanismes, les mécanismes extra sont relativement préférés aux mécanismes contractuels soient un peu plus privilégiés que ceux dit contractuels. Ce qui corrobore les résultats de Peillon (2001) pour lesquels, les mécanismes extra-contractuels s'inscrivent dans la continuité des mécanismes contractuels et viennent en complément de ceux-ci dans l'optique de répondre aux insuffisances des théories contractuelles.

Toutefois, ces mécanismes ne suppriment pas toute forme d'incertitude. II arrive même, qu'ils produisent des effets contraires devant certaines relations. Les analyses du CAS 2 démontrent la difficulté de se prémunir des comportements opportunistes de certains de partenaires malgré la mobilisation des mécanismes.

\section{Vi. Conclusion}

Pour conclure notre recherche, nous faisons une synthèse de la façon dont la problématique a été développée et la démarche empirique effectuée. Ce qui permet de mettre en exergue les apports de la recherche, les limites de la recherche et les chantiers de recherche future.
Le problème central de cette recherche est celui de l'identification et de la classification des mécanismes de prévention de risques au sein des relations interentreprises dans un contexte de pays en développement. La question centrale posée étant celle de savoir comment les PME se prémunissent-elles des risques liés aux relations interentreprises (Relations Interentreprises) ? Pour répondre à cette préoccupation nous avons commencé par présenter les différents risques auxquels sont exposées ces PME, par la suite, nous nous sommes attelé à identifier les mécanismes et enfin nous avons procédé à un classement de ces mécanismes en fonction de leur poids.

Sur la base d'une étude réalisée auprès de quatre entreprises du secteur, nous avons identifié les mécanismes les plus significatifs mis en œuvre par ces entreprises: la confiance, l'engagement et le partage d'information auxquels, il faudra également signaler l'émergence de deux nouveaux indicateurs: - l'intuition «rationnalisée » et l'éthique des affaires. II en ressort que ces mécanismes contribuent à la prévention et à la réduction des risques inhérents aux relations interentreprises et surtout permettent aux PME de trouver leur compte dans leurs relations avec les multinationales.

A partir des résultats obtenus, nous faisons le constat que les entreprises ayant constitué notre centre d'intérêt, mobilisent toutes, les mécanismes contractuels et les mécanismes extra-contractuels. Pour ce qui est des mécanismes extra-contractuels, trois cas sur les quatre (Cas 1, Cas 2 et Cas 3) ont opté pour la confiance, l'engagement et la communication et le Cas 4 n'a mis l'accent que sur la confiance et la communication. Outre ces trois mécanismes (confiance, engagement et communication), le Cas 1 et le Cas 3 mobilisent respectivement des mécanismes plus spécifiques: l'Intuition »rationnalisée » et l'Ethique des affaires. Ces deux mécanismes sont tout à fait nouveaux et n'ont, à notre connaissance, pas encore fait l'objet d'investigation.

En somme nous pouvons dire que Ce travail s'inscrit dans le sillage de nombreux autres travaux en management stratégique (Mohamadou, 2014; Cherbib, 2010; Ouédraougo, 2003; Peillon, 2001). Toutefois, sa principale contribution réside dans l'identification et l'intégration des dimensions nouvelles pour les mécanismes de prévention de risques ce qui semble être caractérisé par le contexte de l'étude.

\section{References Références Referencias}

1. Abitbol L. (2012), Initier des coopérations interorganisationnelles dans les démarches d'écologie industrielle et territoriale : une relecture en termes de sociologie de la traduction et de la théorie des objets-frontière, Thèse de docctorat, Université de Lyon 3, 298 pages. 
2. Aliouat B. \& Taghzouti A. (2007), « Alliances stratégiques et création de valeur: Rentabilité, avantage concurrentiel ou légitimité ? Vers une analyse croisée de modèles complémentaires». XVlème Conférence Internationale de Management Stratégique, Montréal, 6-9 juin.

3. Akerlof G. A. (1970), "The Market for "Lemons": Quality Uncertainty and the Market Mechanism ", The Quarterly Journal of Economics, vol. 84, n³, pp. 488-500.

4. Alvarez S. A. \& Barney J. B. (2001), How entrepreneurial firms can benefitt from alliances whith large partners, Academy of Management, vol. 15, $n^{\circ} 1$, pp. 139-148

5. Anderson E. \& Weitz B. (1992), «The use of pledges to build and sustain commitment in distribution channels", Journal of Marketing Research, February, vol. 29, n¹, pp.18-34

6. Assens C. \& Cherbib J. (2010), L'Alliance asymétrique: une stratégie durable?, La Revue des Sciences de Gestion, 243-244 : 3-4, 111-119.

7. Barabel M., Meier O. \& Soparnot R. (2015), Asymmetric alliances between SMEs and large firms in the area of innovation: strategic determinants and cultural effects, Gestion 2000, vol. 31, n 6, pp. 87106.

8. Beamish P.W. \& Killing J. P. (1997), Cooperative Strategies, the New Lexington Press.

9. Beamish P.W. \& Jung J.C. (2005), «The Performance and Survival of Joint Ventures with Asymmetric Parents», International Business Review, pp. 1-16.

10. Blanchot F. (2006), «Alliances et performances: Un essai de synthèse», Cahier de recherche, CREPA, $n^{\circ} 1,43 p$.

11. Blau P.M. (1964), Exchange and Power in Social Life, Transaction Publishers, $352 \mathrm{p}$.

12. Broussoau E. (1989), «L'approche néo institutionnelle des coûts de transaction", Revue Française d'Economie, vol. 4, n4, pp. 123-166.

13. Brulhart F. (2002), « Le rôle de la confiance dans le succès des partenariats verticaux logistiques: le cas des coopérations entre industriels agroalimentaires et prestataires logistiques ", Finance Contrôle Stratégie, vol. 5, n 4, décembre, p. 51-77.

14. Cao M. \& Zhang Q. (2011), Supply chain collaboration: impact on collaborative advantage and firm performance, Journal of Operations Management, 29(3), 163-180.

15. Cherbib J. \& Cheriet F. (2014), L'instabilité des alliances stratégiques asymétriques : une option programmée par la firme multinationale. Revue Internationale de PME. Vol 27, Numéro 2, 2014, p. 15-38.

16. Cheriet F. \& Dikmen L. (2008), contrôle et confiance dans les alliances stratégiques asymétriques: quelles implications pour la performance et l'instabilité de la relation? Proposition d'un modèle intégrateur d'analyse. XVIlème Conférence Internationale de Management Stratégique, Mai 2831, Nice, France.

17. Cheriet F. \& Dikmen-Gorini L. (2013), Contrat ou confiance? effets de la gouvernance sur les performances des alliances stratégiques asymétriques. Revue des Sciences de Gestion, pp.43-51.

18. Cheriet F. (2009), Instabilité des alliances stratégiques asymétriques: cas des relations entre firmes multinationales et entreprises locales agroalimentaires. Thèse de doctorat, montpellier sup agro, 445 pages.

19. Cheriet F. (2016), "Quelles différences de perception des entreprises locales et des firmes multinationales de leurs relations d'alliances stratégiques instables ?," Revue Internationale P.M.E. 292 (2016): 95-118. DOI : 10.7202/1037924ar

20. Christoffersen J. (2013), a review of antecedents of international strategic alliance performance: synthesized evidence and new directions for core constructs, International Journal of Management Reviews, 3(1), 93-102.

21. Chtourou W. \& Laviolette E. M. (2005), Le rôle des réseaux dans la formation des alliances asymétriques: le cas des pme essaimées. xixème conférence de l'aims, angers, 26p.

22. D'lribarne P. (1989), La logique de I'honneur: gestion des entreprises et traditions nationales, Paris-Edition du Seuil.

23. D'rribarne P., Henry A. \& al. (2002), Cultures et mondialisation. Gérer par-delà les frontières, Seuil, 368 pages.

24. Das T. K. \& Teng B. S. (1999), Managing Risks in Strategic Alliances. The Academy of Management Executive, vol 13, n4, pp 50-62.

25. Das T. K. \& Teng B. S., (2001), "A risk perception model of alliance structuring". Journal of International Management, Vol 07, pp 1-29.

26. Delerue H. (2004), Performance des alliances: une gestion duale du risque relationnel, $13^{e}$ Conférence de l'AIMS, Normandie. Vallée de Seine 2, 3 et 4 juin, 1-32.

27. Delerue H. A. \& Simon E. (2005), « Confiance, contrat et degré d'asymétrie dans les relations d'alliance ", Management International, vol. 10, n¹, pp. 51-62.

28. Dussauge P. \& Garrette B. (1997), Les stratégies d'alliance, Editions d'Organisation, 288 pages.

29. Eisenhardt K. (1989), Building theories from case study research. Academy of Management Review, vol. 14, $n^{\circ} 4$, p. 532-550.

30. Fréchet M. (2007), Les difficultés relationnelles dans les partenariats d'innovation : une approche par le 
contrat psychologique, Finance Contrôle Stratégie, $10: 1,125-148$.

31. Gambetta D. (1988), «Can we trust trust? » In D. Gambetta, éd., Trust: Making and Breaking Cooperative Relations, 213-237 Oxford:Blackwell.

32. Gammoudi L. (2006), "Les réclamations dans le domaine des services Business-to-Business»; troisième tutorat collectif en Marketing du réseau ALM.

33. Gao S. \& Zhang Z. (2008), Opportunism and alliance risk factors in asymetric alliances, Eds IEEE, International conference,1-6, DOI : 10, 1109/SOLI

34. Garcia-Canal E., Duarte C. L. \& al. (2002), Accelerating International Expansion through Global Alliances: a Typology of Cooperative Strategies, Journal of World Business 37: 91-107.

35. Garrette B. \& Dussauge P. (1995), Les stratégies d'alliance. Les éditions d'Organisation, Paris, 288 pages.

36. Geringer J. M. \& Hebert L. (1989), « Control and performance of International Joint Ventures ", Journal of International Business Studies, Vol 20, $n^{\circ} 02$, pp. 235-254.

37. Gronroos C., (1990), Approche relationnelle du marketing dans des contextes de service: L'interface marketing et comportement organisationnel, Journal of Business Research, Volume 20, n 1, pp 3-11

38. Gulati R. (1998), Alliances and Networks, Strategic Management Journal 19: 293-317.

39. Hitt M., Ireland R. D. \& Hoskisson R. (2012), Strategic management cases: competitiveness and globalization, Cengage Learning, Boston, 304p.

40. Inkpen A. (2000), a note on the dynamics of learning alliances: competition, cooperation and relative scope, Strategic Management Journal, vol. 21, $\mathrm{n}^{\circ} 7$, p. $775-780$.

41. Jaouen A. (2006), Les stratégies d'alliance des TPE artisanales, Revue Internationale PME, vol 19, n`34, p. 111-136.

42. Kalaignanam K., Shankar V. \& Varadarajan R.P. (2006), « Asymmetric New Product Development Alliances: Win-Win or Win-Lose Partnerships? ", Working Paper, $\mathrm{n}^{\circ} 2$ Institute for the Study of Business Markets, $36 \mathrm{p}$.

43. Killing J. P. (1983), Strategies for Joint Ventures Success, Croom Helm.

44. Klein B., Crawford R.G. \& Alchian A. A. (1978), Vertical integration, appropriable rents, and the competitive contracting process, Journal of Law and Economics, 21(2), October, 297-326.

45. Koenig C. \& Van Wijk G. (1992), "Alliances interentreprises : le rôle de la confiance", in NOELA. (ed.), Perspectives en Management Stratégique, Tome I, Paris, Economica, 305-327.

46. Koenig G. (1993), les théories de la firme, paris, economica.
47. Kogut B. \& Singh H. (1988), the effect of national culture on the choice of entry mode, Journal of International Business Studies: 411-432.

48. Lambe C. J., Spekman R. E. \& Hunt S. D. (2002), Alliance Competence, Resources, and Alliance Success: Conceptualization, Measurement, and Initial Test, Journal of the Academy of Marketing Science, 30, 141-158.

49. Lazaric N., Lorenz E. (1998), "Trust and Organizational Learning During Inter-Firm Cooperation", in Lazaric N., Lorenz E. (eds), Trust and Economic Learning, London, Edward Elgar, 209-26.

50. Lecocq X. (2003), Comportements d'acteurs et dynamique d'un réseau inter-organisationnel: le phénomène des écarts relationnels, Lille, Thèse de doctorat en Sciences de Gestion.

51. Lee C. H., Venkatraman N., Tanriverdi H. \& lyer B. (2010), Complementarity-based hyper competition in the software industry: Theory and empirical test, 1990-2002, Strategic Management Journal, 31(13), $1431-1456$.

52. Lee Y. \& Cavusgil S.T. (2006), Enhancing Alliance Performance: The Effects of Contractual-Based Versus Relational-Based Governance, Journal of Business Research, 59: 8, 896-905.

53. Mayrhofer U. (2007), Les rapprochements d'entreprises: perspectives théoriques et managériales, Management \&amp; Avenir, 14, 8199.

54. Mcgrath, R. G., \& Kim, J. (2013), Innovation, strategy, and hyper competition, in Dodgson, M., \& Phillips, N., The Oxford Handbook of Innovation Management, Oxford University Press: Oxford, 397419.

55. Ménard C. (1994b), "Organizations as Coordinating Devices », Metroeconomica, 45(3), 224-47.

56. Miles M. \& Huberman M. (2003), Analyse des données qualitatives, De boeck, $632 \mathrm{p}$.

57. Minshall T., Mortara L., Valli R. \& Probert D. (2010), « Making "asymmetric" Partnerships Work", Research Technology Management, vol. 53, n³, pp. 53-63.

58. Mohamadou Mindaoudou Z. D. (2017), Asymétries entre partenaires et confiance : le cas des alliances stratégiques entre PME et multinationales, Revue Internationale P.M.E., Vol.30, $n^{\circ} 1$, pp. 57-84.

59. Mohamadou Mindaoudou Z. D. (2014), les alliances stratégiques entre PME et FMN: spécificités et analyse de la performance. Le cas des PME françaises, Thèse de doctorat, Université de Pau et dans Pays de l'Adour, 389 pages.

60. Morgan R. M. \& Hunt S. D. (1994), The commitment-trust theory of relationship marketing, Journal of Marketing, 58, 20-38. 
61. Mohr J. \& Spekman R. (1994), Characteristics of partnership success: partnership attributes, communication behavior, and conflict resolution techniques, Strategic Management Journal, vol.5, $\mathrm{n}^{\circ} 2,135-152$.

62. Montfort I. (1997), Modélisation du choix de partenaires dans la coopération entre PMI. Thèse de doctorat, Université Paris IX.

63. Moorman, RH et Byrne, ZS (2005). Comment la justice organisationnelle affecte-t-elle le comportement de citoyenneté organisationnelle? Dans J. Greenberg et J. Colquitt (Eds.), Handbook of organization justice (pp. 355 380). Mahwah, NJ : Lawrence Erlbaum

64. Nooteboom B., Berger H. \& N.G. Noorderhaven (1997), Effects Of Trust And Governance On Relational Risk, Academy Management Journal, 40, 2, 308-338.

65. Ouedrago A. (2003), Alliances stratégiques dans les pays en développement: spécificité, management et conditions de performances: une étude d'entreprises camerounaises et burkinabées, Thèse de ph.d. en administration. Hec. Université de montréal, 321 pages.

66. Parkhe A. (1993 b), Partner nationality and the structure- performance relationship in strategic alliances, Organization Science, vol 4, pp. 301-324.

67. Peillon S. (2001). Le pilotage des coopérations interfirmes : le cas des groupements de pme. Thèse de doctorat (n.r), saint-étienne, université jean monnet.

68. Pérez L., Florin J. \& Whitelock J. (2012), « Dancing with elephants: The challenges of managing asymmetric technology alliances ", The Journal of High Technology Management Research, vol. 23, n², pp. 142-154.

69. Prashantham S. \& J. Birkinshaw (2008), Dancing with gorillas: How small companies can partner effectively with MNCS, California Management Review, 1, 51, 6-23.

70. Puthod D., (1995), «Entre confiance et défiance: La vigilance au cœur de la gestion des alliances". Gestion 2000, № 02, Mars- Avril. Pp. 111- 129.

71. Puthod D., (1998), « L'alliance, une option stratégique permettant de contourner les dilemmes classiques de la PME », In Torres O., (Ed) PME : De nouvelles approches, Economica. Paris, p. 95-104.

72. Salk J. E. \& Shenkar O. (2001), Social Identities in an International Joint Venture: An Exploratory Case Study. Organization science 12 (2): 161-178.

73. Sapienza A., Stork D. (2001), Leading biotechnology alliances, Willey-Liss, eds.

74. Sawers J. L., Pretorius M. W. \& Oerlemans L. A. G. (2008), "Safeguarding SMEs dynamic capabilities in technology innovative SME-large company partnerships in South Africa », Technovation, vol. 28, n4, pp. 171-182.

75. Schaan J.-L. (1988). How to Control a Joint Venture Even as a Minority Partner?, Journal of General Management 14(1): 4-16.

76. Seppänen R., Blomqvist K. \& Sundqvist S. (2007), « Measuring inter-organizational trust- a critical review

77. of the empirical research in 1990-2003 ", Industrial Marketing Management, vol. 36, n², pp. 249-265.

Simonin, B. L., \& Gates, R. (2013). Learning from alliance successes and failures of small technology-

78. based firms: a longitudinal study. Journal of International Management Studies, 13(2), 5-28.

79. Williamson O.E. (1985), The economic institutions of capitalism, The Free Press.

Yan A., (1998), "Structural stability and reconfiguration of international joint ventures",

80. Journal of International Business Studies, Vol 29: $n^{\circ}$ 4, pp. 773-795.

Yan, A. \& Gray B. (1994), Bargaining Power, Management Control, and Performance in United States - China Joint Ventures: a Comparative Case Study, Academy of Management Journal 37(6): pp. 1478-1517. 